\title{
Microfluidic dialysis using photo-patterned hydrogel membranes in PDMS chips
}

\author{
Hoang-Thanh Nguyen, Morgan Massino, Camille Keita, and Jean-Baptiste Salmon \\ CNRS, Solvay, LOF, UMR 5258, Univ. Bordeaux, F-33600 Pessac, France.
}

\begin{abstract}
We report the fabrication of permeable membranes for microfluidic dialysis applications in poly(dimethylsiloxane) (PDMS) channels. A maskless UV projection device was used to photo-pattern long hydrogel membranes $(\mathrm{mm}-\mathrm{cm})$ with a spatial resolution of a few microns in PDMS chips integrating also micro-valves. We show in particular that multi-layer soft lithography allows one to deplete oxygen from the PDMS walls using nitrogen gas flow and therefore makes possible in-situ UV-induced polymerization of hydrogels. We also report a simple surface modification of the PDMS channels leading to strongly anchored hydrogel membranes that can withstand trans-membrane pressure drops up to 1 bar without leakages. We then measured the Darcy permeability of these membranes and estimated their cut-off by measuring the kinetics of diffusion of macromolecules of different sizes through the membrane. Finally, we illustrate the opportunities offered by such microfluidic chips for dialysis applications by observing in real time the crystallization of a model protein in a chamber of a few nanoliters.
\end{abstract}

\section{INTRODUCTION}

In recent years, many studies have reported the possibility of integrating membranes in microfluidic systems for various applications such as purification/concentration and separation of samples for (bio-)analytical chemistry [1], chemical engineering [2-4], soft matter [5, 6], desalination [7], etc. In particular, nanoporous membranes are attracting a great deal of interest due to the many applications in the field of dialysis and ultra-filtration. Many works integrate a commercial dialysis membrane by sandwiching it between two microfluidic chips, with applications as diverse as the study of phase diagrams of colloidal systems [8], protein crystallization [9], or to investigate osmotically-driven flows [10] and fouling in ultra-filtration processes [11]. But it is in the field of analytical biochemistry that this type of technique is of particular interest with numerous applications such as on-chip microdialysis sample cleanup, fractionation, concentration, separation, etc, see e.g. [12-17] and the review [18].

Beyond this mechanical technique of membrane integration, in-situ membrane fabrication by interfacial polymerization $[19,20]$ or by photo-patterning offers many advantages [21]. This technique makes it possible in particular to integrate a membrane perpendicularly in a microfluidic channel and not as a distinct layer above or below the channel network. In-situ photo-patterning consists in photo-polymerizing specific mixtures containing monomers (or oligomers), crosslinkers, and a photo-initiator, directly inside a microfluidic channel. Exposure to UV light triggers free-radical polymerization leading to the formation of membranes, either dense and permeable, or nanoporous when porogens are added to the formulation to induce a microscopic phase separation. This technique leads to the integration of membranes with a wide range of nanoporosities in microfluidic chips, and as above, mainly for applications dealing with microdialysis steps of biological samples and biomolecules (concentration, purification, buffer exchange, etc.) [22-28]. Several groups recently focused on poly(ethylene glycol) diacrylate oligomers (PEGDA) as these precursors lead to permeable hydrogels that can be photo-patterned using microscope projection photolithography at a high resolution, down to a few microns [2931]. Squires et al. in particular have extensively used such hydrogel membranes to study colloid diffusio-phoresis at the microfluidic scale $[32,33]$. More recently, Decock et al. [34] also showed that highly permeable nano-porous hydrogel membranes can be obtained in the same way but using porogens in the formulation [35], therefore paving the way for microfluidic studies of processes ranging from ultra- to microfiltration.

The works cited above have demonstrated the successful integration of hydrogel membranes in microfluidic chips made of various materials such as PEGDA, glass, and thiolenebased resists, while poly(dimethylsiloxane) (PDMS) would offer many other possibilities, such as the integration of micro-valves and pumps [36]. The main challenge is the permeability of PDMS to oxygen which inhibits free-radical polymerization [37], thus preventing the polymerization of hydrogels through the whole thickness of the channel and their anchorage on the PDMS walls [38]. Many works even take advantage of this oxygen-permeability to fabricate freestanding hydrogel microstructures using stop flow lithography [39]. Many other works reported the integration of hydrogels inside PDMS chips, mainly for biological applications, see e.g. for stretching DNA [29], biological assays [30], or studies of stem cells in controlled chemical gradients [40]. Nevertheless, the anchoring of the hydrogel to the channel walls is rarely stated, and in-situ fabrication of pressureresistant hydrogel membranes in PDMS devices has never been reported to our knowledge.

In this work, we report a protocol for the fabrication of membranes in PDMS chips for dialysis applications. More precisely, we use a maskless UV projection setup to photopattern PEGDA-based hydrogel membranes of any dimensions and at a high spatial resolution, down to a few microns. To overcome the problems mentioned above, we show that multi-layer soft lithography is a simple technique to perform photo-polymerization in a oxygen-free environment. We also describe a simple silanisation protocol to add vinyl group to the PDMS surface leading to strongly anchored membranes which can withstand trans-membrane pressure drops up to 1 bar. We also report in-situ measurements of their Darcy permeability, as well as kinetics of diffusion of solutes of different molecular weights through the hydrogel membrane. These measurements show that these membranes are particu- 
larly suitable for dialysis applications with a molecular weight cut-off in the range of $\simeq 10-20 \mathrm{kDa}$. We finally illustrate this last point with real-time observations of the crystallization of a model protein by the dialysis method at the microfluidic scale.

\section{MATERIALS AND METHODS}

\section{A. Multi-layer PDMS chips and silanisation protocol}

Multi-layer soft lithography techniques were used to make the PDMS chip shown schematically in Fig. 1a (Sylgard 184, Dow Corning). The bottom layer contains rounded channels (height $h \simeq 20 \mu \mathrm{m}$ ) obtained from a mold made using a positive photoresist (AZ-40XT, AZ Electronic Materials) which is melted and reflowed by heating after the lithography step [36]. The top layer is obtained from a mold made using a negative photoresist (SU-8 3050, MicroChem) and contains rectangular channels (height $H \simeq 85 \mu \mathrm{m}$ ) to close the Quake valves [36] as well as a channel for flowing nitrogen gas to carry out the photo-polymerization in a oxygen-free environment, see below. The fluidic channels are separated from the gas and actuation channels by a thin PDMS layer, $e \simeq 65 \mu \mathrm{m}$. The actuation pressure to close the valves is $\simeq 1.6$ bar and is imposed using a pressure controller (Fluigent, MFCS-EZ). The rounded shape of the fluidic channels is crucial in order to obtain a perfect closing of the valves with the pressure imposed in the actuation channels [36].

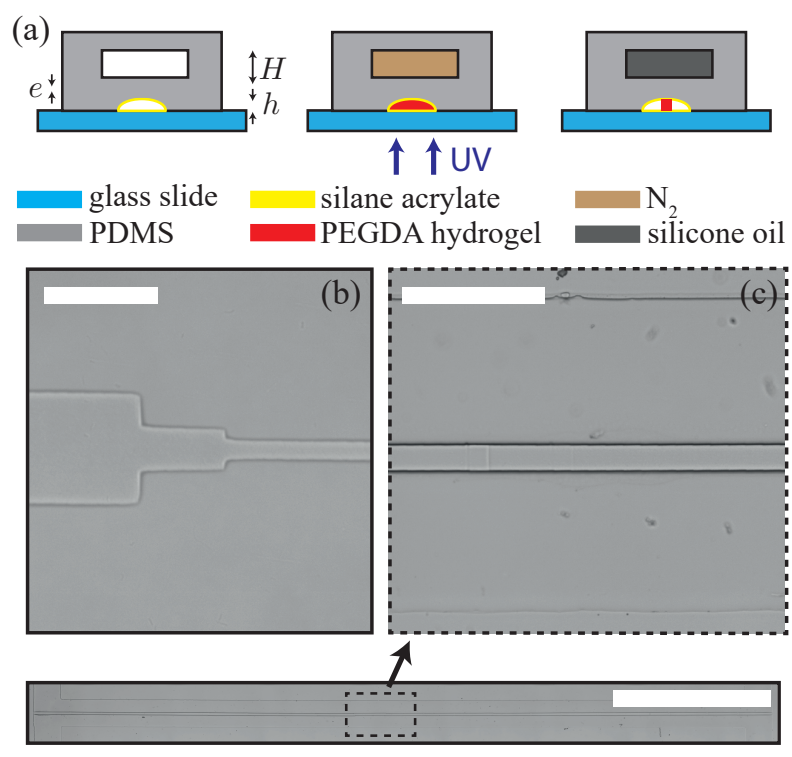

FIG. 1. (a) Schematic protocol for making hydrogel membranes in a multi-level PDMS chip. $H \simeq 85 \mu \mathrm{m}, e \simeq 65 \mu \mathrm{m}$, and $h \simeq 20 \mu \mathrm{m}$. (b) Hydrogel microstructure photo-patterned inside a PDMS channel of height $20 \mu \mathrm{m}$, the smallest width is $10 \mu \mathrm{m}$, scale bar $50 \mu \mathrm{m}$. (c) Long hydrogel membrane $(9.1 \mathrm{~mm})$ of width $25 \mu \mathrm{m}$ inside a PDMS channel (height $20 \mu \mathrm{m}$ ). Scale bars $150 \mu \mathrm{m}$ and $2 \mathrm{~mm}$.

The multi-layer PDMS chip is sealed by a glass slide using a plasma treatment. This step not only ensures covalent anchoring of the PDMS stamp to the glass slide, but also activates the glass and PDMS surfaces for the silanisation step. After plasma, the chip is placed in a oven at $65^{\circ} \mathrm{C}$ for $\simeq 5 \mathrm{~min}$ and pure 3-(trimethoxysilyl)propyl acrylate (SigmaAldrich) is then injected in the fluidic channels to silanize the inner walls. The device is then placed again in the oven for $\simeq 60$ min to promote silanisation and further enhance the PDMS-glass bonding. The channels are finally washed by flowing acetone for several minutes and the chip is placed in a vacuum bell for several hours to remove any possible traces of acetone. Note that we have not observed that this rinsing step with acetone irreversibly changes the shape and size of the channels, although acetone is known to slightly swell PDMS [41].

The integration of the valves as explained in this protocol (rounded channels, alignment with actuation channels of the second layer) is obviously not a mandatory step in the integration of a hydrogel membrane, but allows to combine it with Quake valves for new functionalities, as will be demonstrated later. On the other hand, the alignment of the channel used for the nitrogen flow is not a critical step in the protocol, as it can be significantly wider than the fluidic channels, see for example the masks shown in the ESI $\dagger$, Fig. S1, for making the chips used in this work.

\section{B. Photo-patterning of hydrogel membranes}

The aqueous formulation for the polymerization of the hydrogel membranes is made of PEGDA-700 (average $M_{n}$, Sigma-Aldrich), 2-hydroxy-2-methylpropiophenone for the photo-initiator (Sigma-Aldrich), hydroquinone for the photoinhibitor (Sigma-Aldrich), and water. More precisely, a stock solution of PEGDA/photo-initiator (90/10 \% vol.) containing $23 \mathrm{~g} / \mathrm{L}$ of photo-inhibitor was mixed with water at a volume fraction of 50\%. The same batch of PEGDA formulation was used in all the experiments presented in this work.

For the photo-polymerization of hydrogels of any shape in the channels, we use the Primo device from Alvéole. This setup uses a UV laser diode $(375 \mathrm{~nm})$ combined with a digital micro-mirror device, to project almost-collimated UV patterns (illumination $\simeq 7.7 \mathrm{~mW} / \mathrm{mm}^{2}$ ) at the focal plane of a microscope (IX73 Olympus), with dimensions $\simeq 460 \times 285 \mu \mathrm{m}^{2}$ and spatial resolution of $\simeq 1 \mu \mathrm{m}$ (20X objective, N.A. 0.45 ).

The protocol to photo-pattern membranes in a PDMS channel is shown in Fig. 1a. First, we flow nitrogen gas into the top channel of the chip (pressure drop $\simeq 20$ mbar) causing a rapid depletion of dissolved oxygen in the channel, as well as in the PDMS matrix in the vicinity of the fluid channel, due to the thinness of the PDMS layer between the channels and the high diffusivity of oxygen in the PDMS matrix $\left(D_{\mathrm{O}_{2}} \simeq 3 \times 10^{-9} \mathrm{~m}^{2} / \mathrm{s}[42], e^{2} / D_{\mathrm{O}_{2}} \simeq 1.4 \mathrm{~s}\right)$. Next, the PEGDA formulation is injected to fill the fluidic channels and UV patterns are projected with a density of energy of $10 \mathrm{~mJ} / \mathrm{mm}^{2}$ and exposure time of $\simeq 1.3 \mathrm{~s}$ to polymerize the formulation. Then, the channels are rapidly flushed with water, while the top channel is filled with silicon oil and closed with stoppers to minimize the deformations of the flu- 
idic channels with the applied pressure, see Fig. 1a. Finally, the membrane is extensively flushed with pure water using an imposed trans-membrane pressure drop of $\simeq 250 \mathrm{mbar}$ for $24 \mathrm{~h}$. Performing the photo-patterning in a oxygen-free environment is absolutely crucial. Indeed, we observe that without nitrogen gas, hydrogels do not adhere to the PDMS due to the presence of a thin uncrosslinked layer in the vicinity of the PDMS surface [38].

This protocol combined with the maskless projection setup allows us to fabricate membranes with sharp interfaces and at a high resolution, as clearly evidenced in Fig. 1b showing a hydrogel with a complex shape and transverse dimensions down to $10 \mu \mathrm{m}$. As shown in Fig. 1c, hydrogel membranes with dimensions much larger than the size of the pattern projected by the digital micro-mirror device can also be fabricated by successive steps of photo-polymerization over large dimensions, with a precise alignment of the hydrogel microstructures using a motorized stage (Märzhäuser). During the photo-patterning, the consecutive patterns are slightly superimposed $(\simeq 10-20 \mu \mathrm{m})$ to avoid any leakages at their junction.

\section{Leakage test}

In order to test the robustness of hydrogel membranes made according to the previously explained protocol, we carried out the experiments illustrated in Fig. 2a, see also the movies $\mathrm{M} 1$ and M2 in the ESI $\dagger$. For these experiments, we photo-

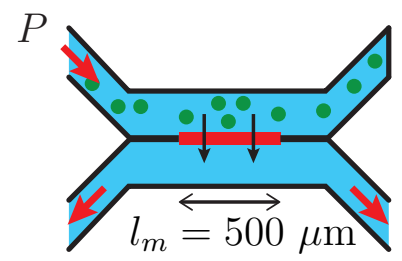

(a)
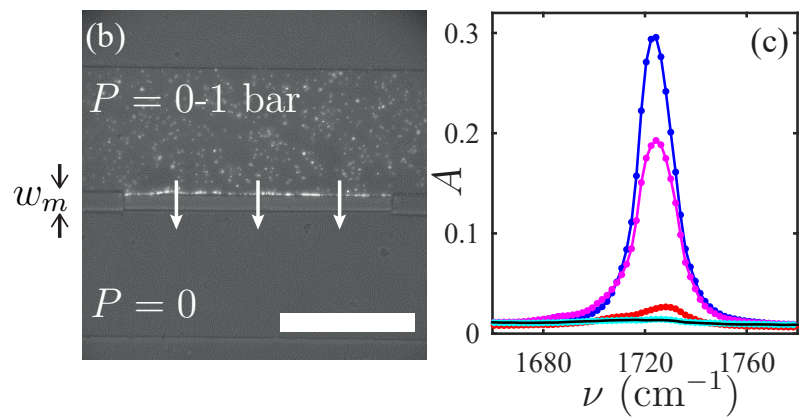

FIG. 2. (a) Experiment to test the tightness of a membrane. (b) Superimposed fluorescence and bright field images showing the accumulation of fluorescent colloids (diameter $1 \mu \mathrm{m}$ ) on the permeable membrane, see the movie M1 in the ESI $\dagger$ (membrane width $w_{m}=25 \mu \mathrm{m}$ ). Scale bar $250 \mu \mathrm{m}$. (c) IR spectra, absorbance $A$ vs. wavenumber $v$. Blue: drop of pure acrylate silane, violet and red: PDMS surfaces treated with the liquid silane before and after the acetone rince step. The cyan spectrum corresponding to a PDMS surface silanized in vapour phase is superimposed with the spectrum of the bare PDMS surface (thin black line). patterned a membrane $\left(l_{m}=500 \mu \mathrm{m}\right.$ long, $w_{m}=25 \mu \mathrm{m}$ wide $)$ in a PDMS chip to separate two channels of height $\simeq 25 \mu \mathrm{m}$ and width $250 \mu \mathrm{m}$. We then injected a highly dilute aqueous dispersion of fluorescently labeled colloids in one of the channels (FluoSpheres, Thermo Fisher Scientific) and closed its outlet using a stopper. The pressure $P$ imposed at the inlet of the channel then causes a flow through the membrane, accumulating colloids on the surface of the membrane. For $P \leq 1$ bar (relatively to the ambient pressure), we did not observe any leakages even with colloids of $20 \mathrm{~nm}$ in diameter, see the movies M1 and M2 in the ESI $\dagger$. At higher pressure, the membrane suddenly detaches irreversibly from the channel walls. These observations show that the surface treatment with the pure liquid silane acrylate leads to strongly anchored membranes on the PDMS and glass surfaces.

The mechanical resistance of a hydrogel membrane depends a priori on its anchoring surface on the glass and PDMS substrates, and therefore on its width and length. The tests described above were carried out with a $l_{m}=500 \mu \mathrm{m}$ long and $w_{m}=25 \mu \mathrm{m}$ wide membrane in order to visualize the whole membrane in the same field of view of the microscope to detect any possible leaks with a high precision. However, it is likely that much thinner and/or much longer membranes may have different pressure rupture thresholds. However, we will show further on that membranes of the same thickness, but much longer $\left(l_{m} \simeq 9 \mathrm{~mm}\right)$, also mechanically resist transmembrane pressure drops of the order of a few hundred mbar. Finally, it should also be noted that the lifetime of a membrane as well as the threshold of mechanical resistance probably also depends on the history of the trans-membrane pressure drops imposed. From our experience, microfluidic devices incorporating long and thin membranes $\left(l_{m} \simeq 9 \mathrm{~mm}, w_{m}=25 \mu \mathrm{m}\right)$ withstood trans-membrane pressures of a few hundred mbar over periods of a few days (see below). However, a quantitative determination of the precise rupture thresholds remains to be carried out according to multiple parameters: duration of the imposed pressures, membrane history, dimensions, etc.

\section{FTIR experiments}

This covalent bonding was further confirmed by ATR-FTIR characterizations from several PDMS surfaces treated with different protocols, see Fig. 2c. More precisely, IR spectra were collected with identical acquisition parameters with a BRUKER Vertex 70 spectrometer associated to a Golden Gate ATR accessory. A DTGS detector was used and 25 scans were collected for each spectrum with a spectral resolution of $4 \mathrm{~cm}^{-1}$. Figure $2 \mathrm{c}$ shows in particular that the surface treatment using the liquid silane acrylate leads to carbonyl groups $\left(v \simeq 1735 \mathrm{~cm}^{-1}\right)$ related to acrylate bonds on the PDMS surface [43], even after the washing step by acetone. On the contrary, these measurements also show that vapour deposition of the same silane (at $65^{\circ} \mathrm{C}$, after plasma treatment) does not lead to any detectable peak. We also observed that silanisation carried out in a vapour phase does not allow efficient anchoring of the hydrogels as leakages and detachment of the membranes are observed at low imposed pressure ( $\simeq 100-200$ mbar). 


\section{RESULTS}

\section{A. Measurements of the Darcy permeability}

The Darcy permeability $\kappa$ of a membrane of thickness $w_{m}$ is defined by the Darcy's law:

$$
v_{m}=\frac{\kappa}{\eta_{w}} \frac{\delta P}{w_{m}},
$$

where $\delta P$ is the trans-membrane pressure drop, $v_{m}$ the transmembrane flow $(\mathrm{m} / \mathrm{s})$, and $\eta_{w}$ the water viscosity. To estimate $\kappa$ for our hydrogel membranes, we performed the experiments shown schematically in Fig. 3a. The chip contains two rounded channels (height $h \simeq 20 \mu \mathrm{m}$, width $150 \mu \mathrm{m}$ ) separated by a long and thin membrane (length $l_{m}=9.1 \mathrm{~mm}$, width $\left.w_{m}=25 \mu \mathrm{m}\right)$. One of the channels is connected to a PTFE tube (Scientific Commodities Inc., inner radius $R=190 \mu \mathrm{m}$, outer radius $550 \mu \mathrm{m}$ ) and its outlet is blocked. The inlet and outlet of the other channel are left opened. The tube is connected to a pressure controller (Fluigent MFCS-EZ) to impose a precise pressure $P$ (relatively to the ambient pressure). The tube is completely filled with water, with the exception of a small air space whose position $X_{m}$ is recorded using a stereo-microscope (SZX10, Olympus). The imposed pressure $P$ leads to a trans-membrane flux through the membrane and thus to a flow rate $Q$ in the tube, displacing the air/water interfaces. To minimize water pervaporation through PDMS, possibly leading to flow rates of the same order of magnitude as $Q$ [44], the chip is fully immersed in a water bath several days prior to the measurements.

In a typical experiment, we imposed constant pressure from $P=350$ mbar to $P=0$, by steps of 50 mbar during about 2 hours, while measuring simultaneously the position $X_{m}$ of the air/water interface along the tube. At each change of pressure level, the air/water interface moves instantaneously by about $200 \mu \mathrm{m}$ (corresponding to a volume of $\delta V \simeq 20 \mathrm{~nL}$ for pressure steps of $\delta P \simeq 50$ mbar), due to the elasticity of the system (air bubble, PDMS channel, tube). As the hydrodynamic resistance $R_{h}$ of the whole setup (tube + PDMS channel) is of the order of $R_{h} \simeq 10^{14} \mathrm{~Pa} \mathrm{~s} / \mathrm{m}^{3}$, the elasto-hydrodynamic time scales $\tau=R_{h} \delta V / \delta P$ are of the order of only a few seconds, and elasticity plays no role on the meniscus displacement on long time scales. The temporal relative displacements $\delta X_{m}$ for each imposed pressure step are shown in Fig. 3b. These data are well-fitted by linear fits leading to flow rates $Q$ ranging from $\simeq 5$ to $\simeq 40 \mathrm{~nL} / \mathrm{h}$ for $P$ ranging from 0 to $350 \mathrm{mbar}$. Note that such small flow rates cannot be measured by any commercial flowmeter, thus justifying the technique described above. Strikingly, the measured flow rate does not vanish for $P=0$ and is roughly $Q_{0} \simeq 7 \pm 2 \mathrm{~nL} / \mathrm{h}$. We have verified that this offset is not due to the pervaporation of water through the PDMS chip, but to the low permeability of the PTFE tube itself, $30 \mathrm{~cm}$ in length. Indeed, we confirmed this result by performing similar experiments but with only a tube closed by a stopper. The measured flow rate is also in agreement with estimations made using literature values of the permeability of water through PTFE (in the range $0.0045-0.3 \mathrm{~g} \cdot \mathrm{mm} /\left(\mathrm{m}^{2}\right.$ day) [45]). (a)
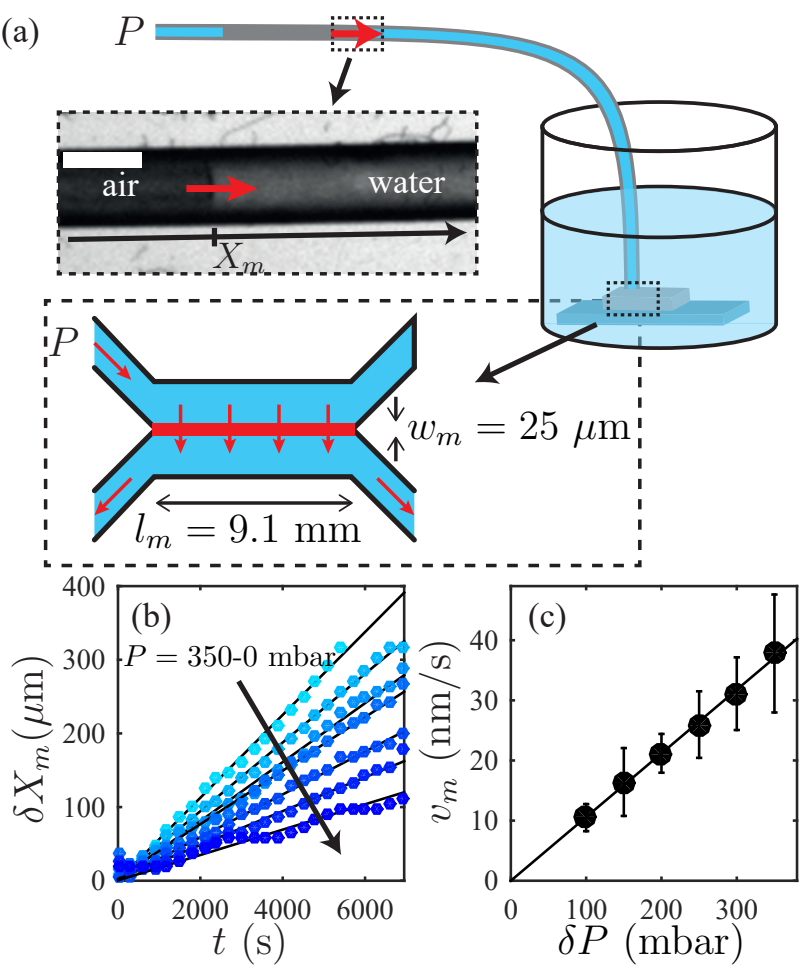

FIG. 3. (a) Measurements of the permeability $\kappa$ of the membrane. Imposed pressure $P$ drives a flow through the membrane. The chip is immersed in a water bath to minimize pervaporation through PDMS. Scale bar $1 \mathrm{~mm}$. (b) Relative displacement $\delta X_{m}$ of the air/water meniscus in the tube for various imposed pressure $P$. (c) Water flux $v_{m}$ vs. trans-membrane pressure drop $\delta P$. Errorbars are calculated from the standard deviation over 4 experiments. The linear fit yields $\kappa \simeq 2.6 \pm 0.5 \times 10^{-20} \mathrm{~m}^{2}$.

In the following, we therefore subtracted this constant offset in order to estimate the trans-membrane flow rate $Q_{m}=Q-Q_{0}$.

For the small flow rates estimated from Fig. 3b, one can fully neglect the pressure drops along the tube and fluidic channels. The trans-membrane pressure drop in eqn (1) is thus homogenous along the membrane and given by $\delta P \simeq P$. Consequently, the trans-membrane flux $v_{m}$ is also homogeneous and related to the trans-membrane flow rate by $Q_{m}=\left(l_{m} h\right) v_{m}$. Figure $3 \mathrm{c}$ shows $v_{m}$ against $\delta P$ calculated from the mean of different experiments performed with several membranes photo-polymerized in similar conditions (errorbars show the standard deviation of these data). These data are well-fitted by eqn (1) with $\kappa \simeq 2.6 \pm 0.5 \times 10^{-20} \mathrm{~m}^{2}$. The measured variation $\left( \pm 0.5 \times 10^{-20} \mathrm{~m}^{2}\right)$ is probably the result of multiple uncertainties such as the precise determination of the dimension of the membrane, especially its width, slightly different illumination conditions, or the error associated to the determination of the meniscus velocity. We observed that a higher exposure energy during the photo-polymerization results in significantly less permeable membranes (data not shown), thus suggesting that our conditions of polymerization lead to partially cross-linked hydrogels. As explained in Sec. II B, we made long membranes by successive steps with slightly superimposed patterns to avoid leakages. These superimposed 
regions have thus undergone a double exposure and are a priori less permeable. However, the overall size of the overlaps ( $\simeq 300 \mu \mathrm{m}$ ) should have no significant effect on the permeability of the membrane due to its length $\left(l_{m}=9.1 \mathrm{~mm}\right)$.

The measured permeability $\kappa \simeq 2.6 \pm 0.5 \times 10^{-20} \mathrm{~m}^{2}$ of our hydrogel membranes is slightly higher than the macroscopic measurement made by Ju et al., $\kappa \simeq 1.35 \times 10^{-20} \mathrm{~m}^{2}$, on thick polymerized film $(300 \mu \mathrm{m})$ obtained from the same PEGDA formulation [46]. This difference could probably be explained by the cross-linking density of the hydrogel, as $\mathrm{Ju}$ et al. stated that their membranes were fully polymerized.

\section{B. Kinetics of solute diffusion through the membrane}
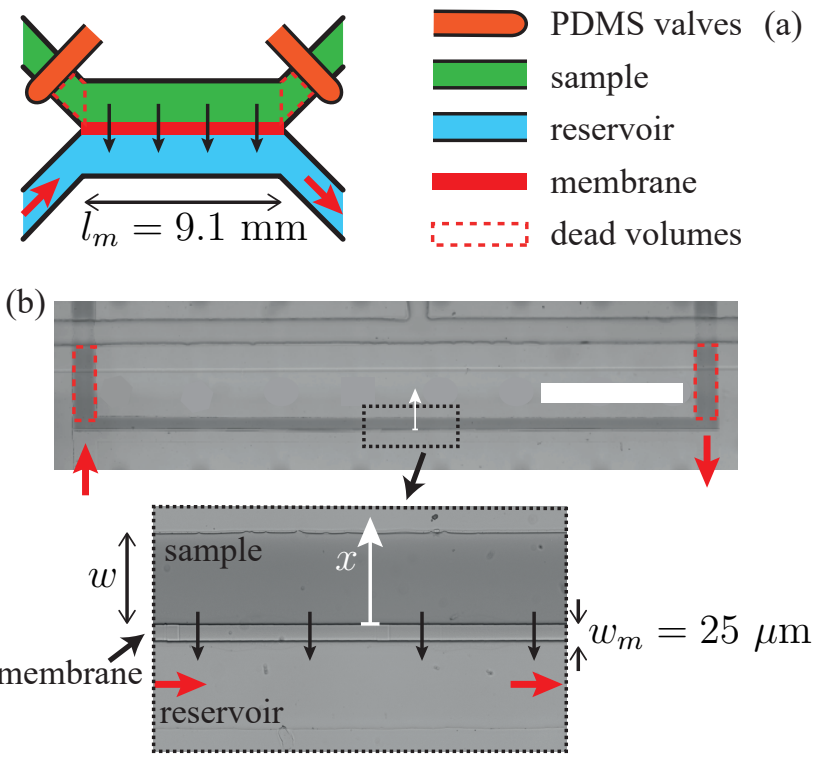

FIG. 4. (a) Schematic view of the microfluidic chip for dialysis applications, see Fig. S1 in the ESI for the masks used for the different layers $\dagger$. (b) Bright field image of the whole chip showing the long and thin hydrogel membrane inside the PDMS channel as well as the two (closed) valves. Dead volumes are highlighted by dotted red lines. Scale bar $2 \mathrm{~mm}$. Inset: zooming in on the central region to better highlight the spatial resolution of the membrane $(w=150 \mu \mathrm{m})$. Molecular dyes (brillant blue FCF, $M_{w} \simeq 790 \mathrm{~g} / \mathrm{mol}$ ) contained in the closed sample chamber diffuse through the membrane into the reservoir channel, see the snapshots in the ESI, Fig. S2 $\uparrow$.

To estimate the molecular weight cut-off of our hydrogel membranes, we performed kinetic measurements of diffusion of molecules of different weights through the membrane of the chip shown in Fig. 4, see also Fig. S1 in the ESI for the masks used for making the different layers of this chip $\dagger$. In this chip designed for dialysis applications, a long and thin hydrogel membrane, $l_{m}=9.1 \mathrm{~mm}$ and $w_{m}=25 \mu \mathrm{m}$, was photopatterned in a PDMS channel (height $h=20 \mu \mathrm{m}$ ) to separate two identical channels of width $w=150 \mu \mathrm{m}$. One of the channels can be closed by two Quake valves, therefore leading to a closed chamber of volume $V_{c} \simeq 38 \mathrm{~nL}$ (estimated using profilometry measurements), referred below to as the sample chamber. The content of this chamber can be modulated by the diffusion of molecules through the membrane from (or to) the other channel called below the reservoir channel.

The first experiments we performed are illustrated in Fig. 4. We first prepared two aqueous solutions of fluorescein with the same concentration $(0.015 \mathrm{mM})$, but with different ethanol contents, 0 and $50 \%$ vol. We then enclosed the aqueous fluorescein solution in the sample chamber, while the $50 \%$ water/ethanol mixture constantly flows in the reservoir channel (imposed pressure drop of 50 mbar). Ethanol and water rapidly interdiffuse through the hydrogel membrane, leading to a change of the fluorescence as the emissivity of the fluorescein molecules depends on the water/ethanol content. The kinetics of the mass transfer is estimated by recording fluorescence images with a $10 \mathrm{X}$ objective using an inverted microscope (Olympus IX83). Typical frame rate is about $10 \mathrm{im}$ ages per minute, with exposure time of $80 \mathrm{~ms}$. We checked in separate experiments that the overall exposure (a few seconds using a shutter) does not lead to significant photo-bleaching with our illumination conditions. This experiment, as well as those presented below, was not carried out by immersing the chip in water, as in the case of permeability measurements. The pervaporation of the water contained in the PDMS chip, and in particular in the sample chamber, therefore leads to a flow of water from the reservoir channel to compensate for the loss of water mass. This mechanism does not change the solute concentration in the chamber, and the very low associated flow rates (at most a few $\mathrm{nL} / \mathrm{h}$ [44]) do not change the measured mass transfer kinetics.

Figure 5a shows the space-time plot of the fluorescence profiles $I_{n}(x, t)$ measured along the $x$ axis shown in Fig. $4 \mathrm{~b}$ at the center of the channel. These data are normalized between 0 and 1 , using the initially homogeneous concentration profile to account for the rounded shape of the channel and the fluorescence intensity of the water/ethanol mixture to set $I_{n}=0$. The fluorescence intensity in the chamber decreases on a time scale $\simeq 20-30$ s with noticeable concentration gradients. The decrease of the fluorescence is due to the interdiffusion of ethanol and water through the hydrogel membrane leading ultimately to a homogeneous $50 \%$ vol. water/ethanol mixture in the chamber. Figure 5b shows the normalized fluorescence intensity measured in the middle of the sample chamber $(x=w / 2=75 \mu \mathrm{m})$ against time. These data are crudely fitted by the exponential decrease $I_{n}(w / 2, t)=\exp \left(-t / \tau_{s}\right)$ with $\tau_{s} \simeq 23 \pm 2 \mathrm{~s}$.

We performed similar experiments with a dilute aqueous fluorescein solution $(0.03 \mathrm{mM})$ to probe the mass transfer of a larger solute (fluorescein molar mass $M_{w}=332 \mathrm{~g} / \mathrm{mol}$ ). In this case, the solution is first injected in the sample channel and isolated by closing the valves. Fluorescein molecules then diffuse through the membrane to the reservoir channel, where they are constantly depleted by a water flow driven by a pressure drop of 50 mbar. As shown by Fig. 5c, concentration profiles $I_{n}(x, t)$ (normalized again to account for the rounded shape of the chamber) remain homogeneous in the chamber in this case and the decrease of the fluorescence intensity at $x=w / 2$ can be again fitted by an exponential decrease but 

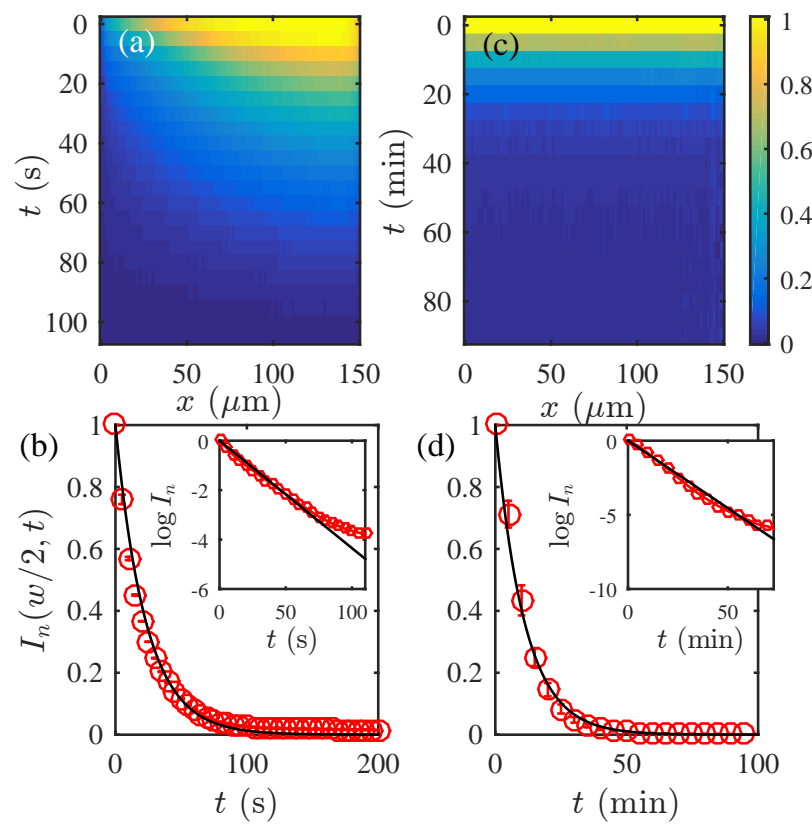

FIG. 5. Space-time plots of the normalized fluorescence profiles $I_{n}(x, t)$ measured along the $x$ axis shown in Fig. 4 for the diffusion through the membrane of ethanol/water (a) and fluorescein (c). $I_{n}(w / 2, t)$ vs. $t$ for the ethanol/water (b) and fluorescein (d) cases. Errorbars are calculated from the standard deviation of 3 experiments. The insets show the same data but in a log-lin scale. The black lines are fits by an exponential decrease with time scales $\tau_{s}=23 \mathrm{~s}$ in (b) and $\tau_{s}=11 \mathrm{~min}$ in (d).

with a much longer time scale, $\tau_{s} \simeq 11 \pm 1 \mathrm{~min}$ (Fig. $5 \mathrm{~d}$ ).

To span a wider range of molecular weights, we performed similar experiments, but with fluorescein isothiocyanate dextrans with molecular weights of $M_{w}=4,10$, and $20 \mathrm{kDa}$, referred below to as FD4, FD10, FD20 (Sigma-Aldrich). Typical concentrations were $0.25,0.1$, and $0.05 \mathrm{mM}$ for FD4, FD10, and FD20 respectively therefore ensuring dilute conditions. For FD4 and FD10, we also observed a homogenous decrease of the fluorescence intensity in the chamber as for the case of fluorescein, yet with much longer times scales $\tau_{s} \simeq$ $110 \pm 10 \mathrm{~h}$ and $\tau_{s} \simeq 120 \pm 10$ days for FD4 and FD10 respectively, see Fig. S3 in the ESI $\dagger$. These time scales were again estimated by fitting the temporal decrease of the fluorescence in the middle of the chamber by $I_{n}(w / 2, t)=\exp \left(-t / \tau_{s}\right)$. The $\tau_{s}$ values are nevertheless associated to large uncertainties, as measurements were only performed during $\simeq 30$ and $\simeq 100 \mathrm{~h}$ for FD4 and FD10 respectively, leading only to a slight decrease of the intensity $\left(I_{n}(w / 2) \simeq 0.7\right.$ for FD4 and $I_{n}(w / 2) \simeq 0.97$ for FD10, see Fig. S3 in the ESI $\left.\dagger\right)$. In the case of FD20, we did not measure any noticeable decrease of the fluorescence even after 3 days in the sample chamber (typical frame rate 10 images per day).

Figure 6 displays the measured time scales $\tau_{s}$ against the molecular weight of the corresponding solute, see also Table I. These data yield a crude estimate of the molecular weight cut-off (MWCO) of our membrane, in the range of 10-20 kDa. Note that our estimate differs from a strict mea- surement of the MWCO traditionally done using retention experiments in the context of membrane science [21]. Nevertheless, our kinetic measurements unambiguously show that the chip shown in Fig. 4 can be used for any dialysis applications on time scales of several days with macromolecules of molecular weight $\geq 10 \mathrm{kDa}$. Note that macroscopic measurements performed by Ju et al. on thick cross-linked hydrogels obtained using the same PEGDA formulation lead to a significantly smaller MWCO, around $1.2 \mathrm{kDa}$ [46]. As for the Darcy permeability, this difference could be due to the difference of cross-linking density, leading to different mesh sizes of the hydrogel network. This discrepancy could also be due to the difference between the methodology used by Ju et al. to measured the cut-off (retention of PEG macromolecules in a dead-end filtration experiment) and our kinetic measurements of a purely diffusive mass transfer.

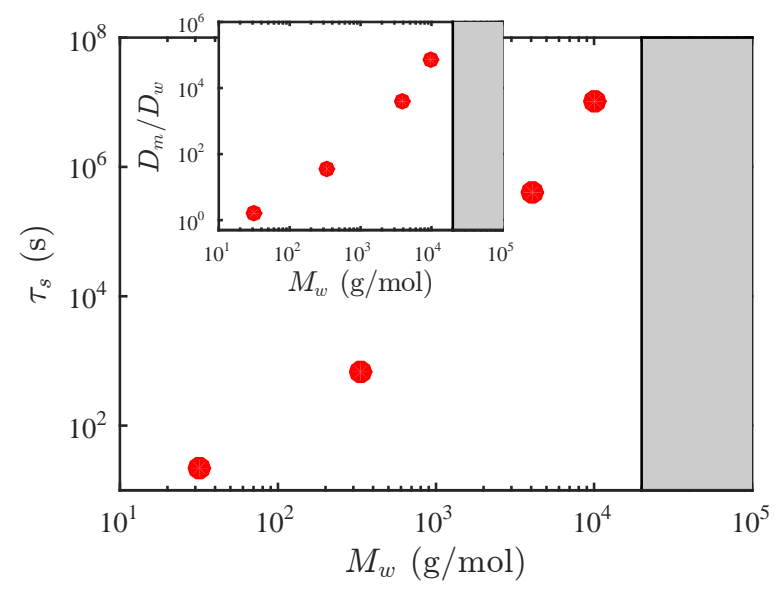

FIG. 6. $\tau_{s}$ vs. molar mass $M_{w}$ of the different solutes studied, see also Table I. The inset shows $D_{w} / D_{m}$ vs. $M_{w}$ assuming $P_{a}=0.5$, see text. The gray area indicates the range of molar mass for which solutes do not cross the membrane on the time scale of the measurements ( 23 days $)$.

TABLE I. Values of molecular weight $M_{w}$, diffusion coefficient in water $D_{w}, \tau_{s}, \tilde{\tau}_{s}, \Re_{m} / \Re_{w}$, and $D_{m} / D_{w}$ (assuming $P_{a}=0.5$ ) for the different solutes studied. For the ethanol/water case, the water content varies from 0.5 to 1 and $D_{w} \simeq 4-12 \times 10^{-10} \mathrm{~m}^{2} / \mathrm{s}$ [47]. We chose the average value $D_{w}=8 \times 10^{-10} \mathrm{~m}^{2} / \mathrm{s}$ for simplicity and we used the average molar mass $M_{w}=32 \mathrm{~g} / \mathrm{mol}$.

\begin{tabular}{lllll}
\hline Solutes & water/ethanol & fluorescein & FD4 & FD10 \\
\hline$M_{w}(\mathrm{~g} / \mathrm{mol})$ & 32 & 332 & 4000 & 10000 \\
$D_{w} \times 10^{10}\left(\mathrm{~m}^{2} / \mathrm{s}\right)$ & $8[47]$ & $4.2[48]$ & $1.1[49]$ & $0.8[49]$ \\
$\tau_{s}(\mathrm{~s})$ & 23 & 660 & $4 \times 10^{5}$ & $1 \times 10^{7}$ \\
$\tilde{\tau}_{s}$ & 0.8 & 12.3 & 2000 & 37000 \\
$\Re_{m} / \Re_{w}$ & 0.45 & 11.6 & 1350 & 25000 \\
$D_{w} / D_{m}$ & 1.3 & 35 & 4000 & 75000 \\
\hline
\end{tabular}




\section{Mass transfer kinetics: role of the dead volumes}

As demonstrated above, the chip shown in Fig. 4 can be used for dialysis applications with macromolecules of molecular weight $\geq 10 \mathrm{kDa}$. The same concept was previously demonstrated by Paustian et al. [31] with a similar design integrating also hydrogel membranes in a NOA chip, yet with a smaller membrane length $\left(900 \mu \mathrm{m}\right.$ vs. $\left.l_{m}=9.1 \mathrm{~mm}\right)$ and valves located outside the chip. Mass transfer is mainly governed in such experiments by two processes: (i) solute diffusion in the sample channel with resistivity $\mathfrak{R}_{w}=w / D_{w}$ and (ii) solute diffusion through the membrane with resistivity $\mathfrak{R}_{m}=w_{m} /\left(P_{a} D_{m}\right)$ [31]. In the above relations, $D_{w}, D_{m}$ are the diffusion coefficients of the solute in the solution and in the hydrogel respectively and $P_{a}$ is the partition coefficient defined by the ratio between the equilibrium concentrations in the hydrogel membrane and in the solution, $P_{a}=C_{m} / C$. Nevertheless, the sample chamber shown in Fig. 4 has also dead volumes for obvious geometrical constraints (i.e. volumes that are not in direct contact with the membrane), which can also influence the overall kinetics of mass transfer.

In order to get more insights into the role of the dead volumes and of the resistivity $\mathfrak{R}_{w}$ and $\mathfrak{R}_{m}$, we consider theoretically the following case mimicking the experiments performed above: a dilute solution at an initial solute concentration $C_{0}$ enclosed in the sample chamber and a flow of the pure solvent in the reservoir channel, large enough to impose $C=0$ at the membrane surface in the reservoir. We then performed numerical resolutions of the solute conservation equation for this experimental case, but with the simplified 2D geometry shown in Fig. 7a. For the sake of simplicity, we solved the following $2 \mathrm{D}$ diffusion equation:

$$
\frac{\partial C}{\partial t}=D_{w}\left(\frac{\partial C}{\partial x^{2}}+\frac{\partial C}{\partial y^{2}}\right)
$$

where $C(x, y)$ is the solute concentration in the chamber, assuming homogeneous concentration over the channel height and omitting the rounded shape of the channel. After a transient of the order of $\sim w_{m}^{2} / D_{m}$ (for $P_{a} \leq 1$ ), one can assume quasisteady diffusive permeation [31], leading to the following condition for the solute flux at the membrane surface:

$$
D_{w}\left(\frac{\partial C}{\partial x}\right)_{x=0, y, t}=P_{a} \frac{D_{m}}{w_{m}} C(x=0, y, t) .
$$

On the other boundaries, we simply impose solute no-flux conditions:

$$
D_{w} \nabla C . \mathbf{n}=0
$$

with $\mathbf{n}$ the unit normal vector. Eqn (2)-(4) can be made dimensionless, defining $\tilde{t}=t D_{w} / w^{2}, \tilde{x}=x / w, \tilde{y}=y / w$, and $c=C / C_{0}$ leading finally to:

$$
\begin{aligned}
& \frac{\partial c}{\partial \tilde{t}}=\frac{\partial c}{\partial \tilde{x}^{2}}+\frac{\partial c}{\partial \tilde{y}^{2}} \\
& \left(\frac{\partial c}{\partial \tilde{x}}\right)_{\tilde{x}=0, \tilde{y}, \tilde{t}}=\frac{\mathfrak{R}_{w}}{\mathfrak{R}_{m}} c(\tilde{x}=0, \tilde{y}, \tilde{t}) \\
& \nabla c . \mathbf{n}=0 \text { (solid boundaries) }
\end{aligned}
$$

evidencing the crucial role played by the ratio $\Re_{m} / \Re_{w}$ on the overall mass transfer. These equations were solved numeri-

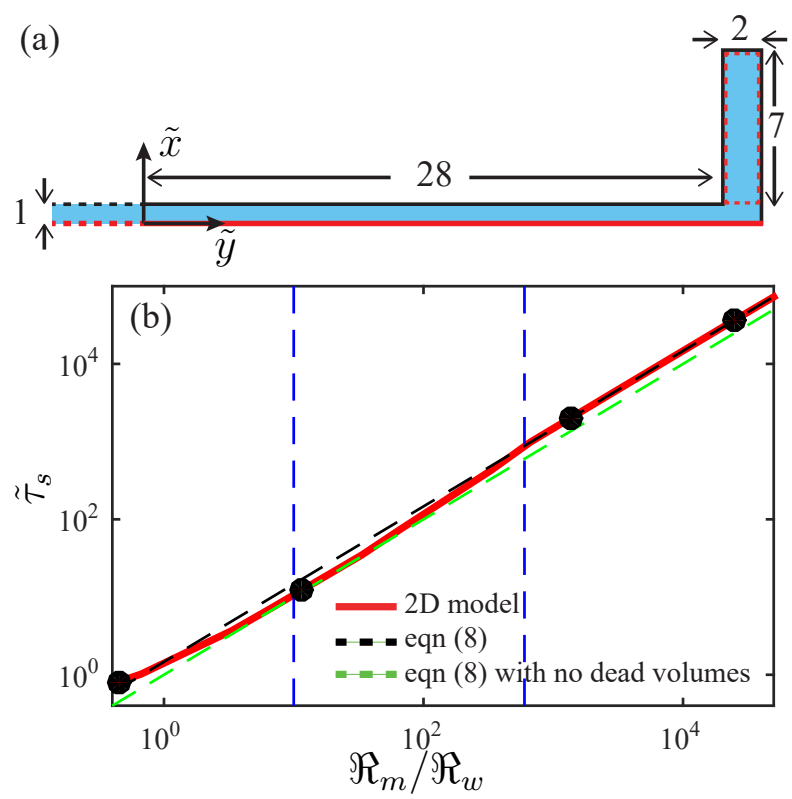

FIG. 7. (a) Computing domain of the dimensionless model for solute diffusion inside the sample channel. The red line is the hydrogel membrane with the boundary condition eqn (6) and the dead volume is highlighted by a dotted red line (only half of the chip is shown). (b) $\tilde{\tau}_{s}$ vs. $\Re_{m} / \mathfrak{R}_{w}$ from the $2 \mathrm{D}$ model (red line). The dashed black line corresponds to the assumption of homogeneous concentration in the chamber, eqn (8). The green line corresponds to the hypothesis of homogeneous concentration without dead volumes $\tilde{\tau}_{s}=\Re_{m} / \Re_{w}$. The black dots are the experimental data given in Table I. The vertical dashed lines located at $\Re_{m} / \Re_{w}=10$ and $\Re_{m} / \Re_{w}=600$ delineate the different regimes.

cally (partial differential equation toolbox, Matlab R2017b) on the domain shown in Fig. 7a using the initial condition $c(t=0)=1$ for various $\mathfrak{R}_{m} / \mathfrak{R}_{w}$ ranging from 0.1 to $10^{5}$. The dimensions of the computational domain have been chosen to correspond approximately to those of the actual chip shown in Fig. 4.

These numerical results help to evidence different kinetic regimes, see the $2 \mathrm{D}$ maps in Fig. S4 in the ESI $\dagger$. For $\mathfrak{R}_{m} / \mathfrak{R}_{w} \gg 600$, the concentration decreases homogeneously within the whole chamber down to $c=0$. For $10 \ll$ $\mathfrak{R}_{m} / \mathfrak{R}_{w} \ll 600$, the concentration profiles decrease almost homogeneously only along the membrane, as concentration gradients persist over longer time scales in the vicinity of the dead volumes. For smaller $\mathfrak{R}_{m} / \mathfrak{R}_{w}$, noticeable concentration gradients are evidenced along $\tilde{x}$, even at the center of the channel $(\tilde{y}=0)$. These 2D numerical results also help us to reveal that the concentration in the middle of the chamber is wellapproximated over the range of investigated $\Re_{m} / \Re_{w}$ by an exponential decay $c(\tilde{x}=1 / 2, \tilde{y}=0, t) \simeq \exp \left(-\tilde{t} / \tilde{\tau}_{s}\right)$, see Fig. S4 in the ESI $\dagger$.

Figure $7 \mathrm{~b}$ shows the estimated $\tilde{\tau}_{s}$ vs. $\mathfrak{R}_{m} / \mathfrak{R}_{w}$ on a log$\log$ scale, evidencing the crucial role played by $\mathfrak{R}_{m} / \mathfrak{R}_{w}$ on the kinetics. Assuming a homogeneous concentration over the 
chamber, integration of eqn (5)-(7) over the domain strictly leads to an exponential decrease with the time scale:

$$
\tilde{\tau}_{s}=\frac{\tilde{S}}{\tilde{l}_{m}} \frac{\mathfrak{R}_{m}}{\mathfrak{R}_{w}},
$$

where $\tilde{l}_{m}$ is the dimensionless length of the membrane and $\tilde{S}$ the dimensionless surface of the chamber $\left(\tilde{S} / \tilde{l}_{m} \simeq 1.47\right.$ in our 2D geometry). Figure $7 \mathrm{~b}$ shows this analytical approximation, as well as the approximation assuming negligible dead volumes, i.e. $\tilde{\tau}_{s}=\mathfrak{R}_{m} / \mathfrak{R}_{w}$. These results help us again to evidence the different regimes discussed above. In particular, it demonstrates that the kinetics far from the dead volumes can be approximated by a simple 1D diffusion problem for $\mathfrak{R}_{m} / \mathfrak{R}_{w} \ll 600$. For larger ratios of resistivity, $\Re_{m} / \Re_{w} \gg 600$, dead volumes also play a role and concentration decreases homogeneously within the whole chamber. Note that for the actual chip shown in Fig. 4, dead volumes are relatively small compared the volume of the chamber, $V_{c} \simeq 38 \mathrm{~nL}$, because our microfabrication protocol makes it possible to integrate micro-valves within the PDMS chip. More precisely, the ratio $\tilde{S} / \tilde{l}_{m}$ in eqn (8) can be estimated in the actual geometry by integrating eqn (2)-(4) and assuming homogeneous concentration leading to

$$
\tilde{\tau}_{s}=\frac{V_{c}}{h l_{m} w} \frac{\Re_{m}}{\Re_{w}},
$$

and thus

$$
\tau_{s}=\frac{V_{c}}{h l_{m} w} \frac{w w_{m}}{D_{m} P_{a}} .
$$

The dimensions of the actual device yield $V_{c} /\left(h l_{m} w\right) \simeq 1.4$, showing that a simple 1D model (i.e. neglecting the dead volumes) should roughly approximate the global kinetics, even for a large resistivity ratio.

All these numerical results are in line with our experimental observations on the diffusion of water/ethanol mixtures and of larger solutes (fluorescein and fluorescently labeled dextrans), see in particular the space-time plots of Fig. 5, but also the extended views, Fig. S2 in the ESI $\dagger$, showing the transport of a molecular dye of molar mass $M_{w} \simeq 790 \mathrm{~g} / \mathrm{mol}$ (brillant blue FCF) in the dialysis chip. One can go a step further and use the experimental values $\tau_{s}$ to estimate the dimensionless time scale $\tilde{\tau}_{s}$ and therefore the ratio $\mathfrak{R}_{m} / \mathfrak{R}_{w}$ using Fig. $7 \mathrm{~b}$. These values are listed in Table I for the different solutes investigated (see also the black dots in Fig. 7b). It could also be tempting to extract from these data the ratio $D_{w} / D_{m}$ assuming a constant partition coefficient $\left(P_{a} \simeq 0.5\right.$ in our case, the hydrogel water volume fraction), valid for negligible interactions between the solutes and the hydrogel network and negligible size exclusion effects [50].

The inset of Fig. 6 displays $D_{w} / D_{m}$ vs. the molecular weight $M_{w}$ of the investigated molecules, see also Table I. For the ethanol/water interdiffusion, we found $D_{w} / D_{m} \sim 1$ demonstrating that the hydrogel hardly affects the diffusivity of these small molecules. For larger molecules, $D_{w} / D_{m}$ strongly increases with $M_{w}$ due to hindered diffusion through the hydrogel network [51-53]. It may also be tempting to compare these data with different models of molecular transport in hydrogels, see e.g. [54, 55], but such a comparison would require careful measurements of the partition coefficient $P_{a}$ (assumed above constant), as $P_{a}$ is expected to decrease significantly due to size exclusion effects, in particular for the polymers investigated above [50].

\section{Application: protein crystallization}

To illustrate the opportunities offered by the integration of valves and a membrane inside a PDMS microfluidic chip, we used the device shown in Fig. 4 to perform real-time observations of the crystallization of a model protein by the dialysis method. More precisely, we prepared lysozyme solutions (chicken egg white, HR7-110, Hampton Research) at $30 \mathrm{mg} / \mathrm{ml}$ in a $100 \mathrm{mM}$ sodium acetate buffer at $\mathrm{pH}$ 4.0. We also prepared an aqueous solution of crystallyzing agents, $\mathrm{NaCl}(7 \mathrm{wt} \%)$ also in a $100 \mathrm{mM}$ sodium acetate buffer at $\mathrm{pH}$ 4.0 .

First, the protein solution was injected in the sample channel and the Quake valves were closed. The crystallizing solution was then injected in the reservoir channel with an imposed pressure drop of 50 mbar. Real-time observations were performed with bright-field microscopy of the sample channel, see Fig. 8a. The lysozyme molecules (molecular weight $M_{w}=14.6 \mathrm{kDa}$ ) are not expected to diffuse through the membrane over the timescale of the experiments, see Fig. 6 . On the other hand, salt molecules do diffuse rapidly through the membrane leading to a supersaturated solution in which protein crystals form and grow [56], see Fig. 8b and the movie M3 in the ESI $\dagger$. Crystals that nucleated in the sample chamber reached their maximal size $(\simeq 25 \mu \mathrm{m})$ within about $30 \mathrm{~min}$. This time scale is much longer than the expected time scale of the diffusion of salts and the crystallization kinetics is therefore not limited by the mass transfer through the membrane, see the horizontal and vertical lines $\mathrm{A} \rightarrow \mathrm{B}$ and $\mathrm{B} \rightarrow \mathrm{C}$ in the solubility diagram shown in Fig. 8c. This rapid mass transfer kinetics (due to the thinness of the membrane and the microfluidic scale) is an important advantage compared to other conventional methods such as vapour diffusion and it offers the possibility of fine-tuning the trajectories explored in a phase diagram.

To illustrate the possibility of dynamic investigations, we then flowed a $\mathrm{NaCl}$ solution at a lower concentration ( $2 \mathrm{wt} \%$ in a $100 \mathrm{mM}$ sodium acetate buffer at $\mathrm{pH} 4.0$ ) to dissolve the crystals, see the movie M3 in the ESI $\dagger$ and the trajectory $\mathrm{C} \rightarrow \mathrm{D}$ in the diagram of Fig. 8c. Before reaching the complete dissolution expected for this salt concentration [57], we again imposed a higher concentration of salt $(7 \mathrm{wt} \%)$ to make the crystals grow again and nucleate new ones, trajectory $\mathrm{D} \rightarrow \mathrm{E} \rightarrow \mathrm{C}$. This simple result shows the capability to perform complex kinetic explorations within the phase diagram of a protein confined in a nanoliter chamber. 


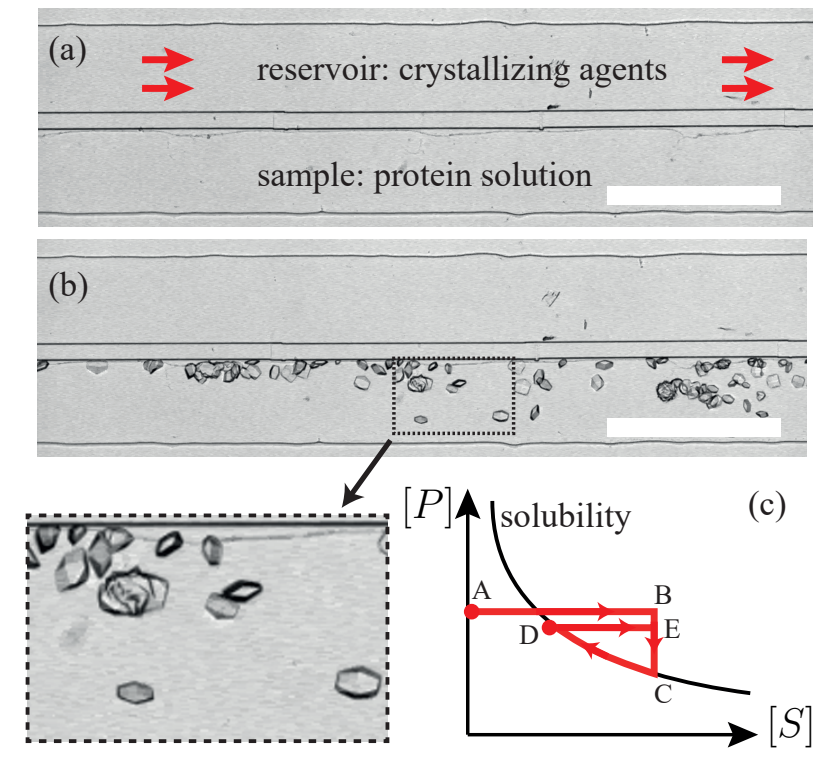

FIG. 8. (a) First moments of the experiment: a protein solution is trapped in the sample chamber, while salts (crystallizing agents) diffuse through the membrane from the reservoir channel (membrane width $25 \mu \mathrm{m}$, scale bar $300 \mu \mathrm{m}$ ). (b) Protein crystals grow and reach their maximum size in about $30 \mathrm{~min}$. (c) Sketch of the kinetic exploration shown in the video M3 provided in the ESI $\dagger$ within the solubility diagram, protein concentration $[P]$ vs. salt concentration $[S]$.

\section{CONCLUSIONS}

In this work, we presented a method to fabricate thin hydrogel membranes with possibly large dimensions $(\mathrm{mm}-\mathrm{cm})$ in PDMS channels. The fabrication protocol is based on standard techniques (multi-layer soft lithography, silanisation, microscope projection photolithography) and leads to membranes which easily withstand trans-membrane pressure drops up to 1 bar. In addition, we also performed a thorough investigation of the mass transfer through such membranes (solvent permeability and kinetics of diffusion of solutes of different weights), showing that these membranes are particularly suitable for any dialysis application with a molecular cut-off in the
10-20 kDa range. We also reported a microfluidic chip combining Quake valves and a long hydrogel membrane to perform dialysis in a nanoliter enclosed chamber and illustrated its functioning using dynamic crystallization experiments of a model protein. The possibility to integrate directly the valves within the channel, in the vicinity of the membrane, is also a strong asset regarding the ratio membrane surface/chamber volume (negligible dead volumes).

In a related work [34], we recently reported the fabrication of pressure-resistant nano-porous hydrogel membranes using a similar method but in PEGDA chips and with porogens added in the hydrogel formulation (PEG) [35]. The Darcy permeability of such membranes is several orders of magnitude higher than the one measured here without porogens $(\kappa \simeq 1$ $10 \times 10^{-17} \mathrm{~m}^{2}$ vs. $\kappa \simeq 2.6 \pm 0.5 \times 10^{-20} \mathrm{~m}^{2}$ ). We also made such highly permeable membranes in our PDMS chips with the protocol explained above and demonstrated that the latter also withstand large trans-membrane pressure drops (data not shown). Our methodologies thus offer the possibilities to integrate membranes in PDMS chips to investigate processes ranging from dialysis to ultra- and micro-filtration (with moderate trans-membrane pressure drops $<1$ bar), with the versatility of PDMS devices, such as the integration of valves and pumps. We hope in the near future to use such unique tools to probe mass transfer within complex fluids.

\section{CONFLICTS OF INTEREST}

There are no conflicts to declare.

\section{ACKNOWLEDGEMENTS}

We would like to thank the exchange program with the University of Lehigh and in particular D. Ou-Yang, for the visit of M. Massimo in our laboratory. We thank J. Jolly for his help for the FTIR experiments, V. Studer and A. Pasturel for scientific exchanges concerning the photo-patterning of hydrogels, Y. Hallez, M. Meireles, and P. Bacchin for discussions concerning the characterization of the membranes. The authors also thank ANR OSMOCHIP (ANR-18-CE06-0021), as well as Solvay and CNRS for funding.
[1] L. Moskvin and T. Nikitina, Journal of Analytical Chemistry, 2004, 59, 2.

[2] I. Ziemecka, B. Haut and B. Scheid, Lab Chip, 2015, 15, 504.

[3] Y. Zhang, N. E. Benes and R. G. H. Lammertink, Chemical Engineering Journal, 2016, 284, 1342.

[4] Y. Kaufman, R. Kasher, R. G. Lammertink and V. Freger, J. Membrane Sci., 2012, 396, 67.

[5] J. Scrimgeour, J. K. Cho, V. Breedveld and J. Curtis, Soft Matter, 2011, 7, 4762 .

[6] L. Daubersies, J. Leng and J.-B. Salmon, Lab Chip, 2013, 13, 910.
[7] B. Gumuscu, A. S. Haase, A. M. Benneker, M. A. Hempenius, A. van den Berg, R. G. Lammertink and J. C. Eijkel, Adv. Funct. Mater., 2016, 26, 8547.

[8] M. Kornreich, M. Heymann, S. Fraden and R. Beck, Lab Chip, $2014,14,3700$.

[9] N. Junius, S. Jaho, Y. Sallaz-Damaz, F. Borel, J.-B. Salmon and M. Budayova-Spano, Lab Chip, 2020, 20, 296.

[10] K. H. Jensen, J. Lee, T. Bohr and H. Bruus, Lab Chip, 2009, 9, 2093.

[11] H. Di, G. J. Martin and D. E. Dunstan, J. Membr. Sci., 2017, 532, 68-75. 
[12] N. Xu, Y. Lin, S. A. Hofstadler, D. Matson, C. J. Call and R. D. Smith, Anal. Chem., 1998, 70, 3553.

[13] F. Xiang, Y. Lin, J. Wen, D. W. Matson and R. D. Smith, Anal. Chem., 1999, 71, 1485.

[14] R. C. Kolfschoten, A. E. Janssen and R. M. Boom, Journal of Separation Science, 2011, 34, 1338.

[15] M. Skou, S. Skou, T. G. Jensen, B. Vestergaard and R. E. Gillilan, J. Appl. Cryst., 2014, 47, 1355.

[16] N. To, I. Sanada, H. Ito, G. S. Prihandana, S. Morita, Y. Kanno and N. Miki, Front. Bioeng. Biotechnol., 2015, 3, 70.

[17] F. Li, R. M. Guijt and M. C. Breadmore, Anal. Chem., 2016, $\mathbf{8 8}, 8257$.

[18] P.-C. Wang, D. L. DeVoe and C. S. Lee, Electrophoresis, 2001, 22, 3857.

[19] B. Zhao, N. O. Viernes, J. S. Moore and D. J. Beebe, 2002, 124, 5284-5285.

[20] H. Hisamoto, Y. Shimizu, K. Uchiyama, M. Tokeshi, Y. Kikutani, A. Hibara and T. Kitamori, Anal. Chem., 2003, 75, 350.

[21] J. De Jong, R. G. Lammertink and M. Wessling, Lab Chip, $2006,6,1125$.

[22] S. Song, A. K. Singh, T. J. Shepodd and B. J. Kirby, Anal. Chem., 2004, 76, 2367.

[23] S. Song, A. K. Singh and B. J. Kirby, Anal. Chem., 2004, 76, 4589.

[24] M. F. Bedair and R. D. Oleschuk, Anal. Chem., 2006, 78, 1130.

[25] H. Chun, Electrophoresis, 2018, 39, 2181.

[26] H. Kim, J. Kim, E.-G. Kim, A. J. Heinz, S. Kwon and H. Chun, Biomicrofluidics, 2010, 4, 043014.

[27] R. T. Davies, J. Kim, S. C. Jang, E.-J. Choi, Y. S. Gho and J. Park, Lab Chip, 2013, 12, 5202.

[28] J. Moorthy and D. J. Beebe, Lab Chip, 2003, 3, 62.

[29] G. C. Randall, K. M. Schultz and P. S. Doyle, Lab Chip, 2006, 6, 516.

[30] J. Liu, D. Gao, H. F. Li and J. M. Lin, Lab Chip, 2009, 9, 1301.

[31] J. S. Paustian, R. N. Azevedo, S.-T. B. Lundin, M. J. Gilkey and T. M. Squires, Phys. Rev. X, 2013, 3, 041010.

[32] N. Shi, R. Nery-Azevedo, A. I. Abdel-Fattah and T. M. Squires, Phys. Rev. Lett., 2016, 117, 258001.

[33] R. Nery-Azevedo, A. Banerjee and T. M. Squires, Langmuir, 2017, 33, 9694.

[34] J. Decock, M. Schlenk and J.-B. Salmon, Lab Chip, 2018, 18, 1075.

[35] A. G. Lee, C. P. Arena, D. J. Beebe and S. P. Palecek, Biomacromolecules, 2010, 11, 3316.
[36] M. A. Unger, H. P. Chou, T. Thorsen, A. Scherer and S. R. Quake, Science, 2000, 288, 113.

[37] S. C. Ligon, B. Husar, H. Wutzel, R. Holman and R. Liska, Chem. Rev., 2013, 114, 557.

[38] D. Dendukuri, P. Panda, R. Haghgooie, J. M. Kim, T. A. Hatton and P. S. Doyle, Macromolecules, 2008, 41, 8547.

[39] D. Dendukuri, D. C. Pregibon, J. Collins, T. A. Hatton and P. S. Doyle, Nat. Mater., 2006, 5, 365.

[40] A. Manfrin, Y. Tabata, E. R. Paquet, A. R. Vuaridel, F. R. Rivest, F. Naef and M. P. Lutolf, Nat. Methods, 2019, 16, 640.

[41] J. N. Lee, C. Park and G. M. Whitesides, Anal. Chem., 2003, $\mathbf{7 5}, 6544$.

[42] D. A. Markov, E. M. Lillie, S. P. Garbett and L. J. McCawley, Biomedical microdevices, 2014, 16, 91.

[43] G. Socrates, Infrared and Raman characteristic group frequencies: tables and charts, John Wiley \& Sons, 2004.

[44] G. C. Randall and P. S. Doyle, Proc. Natl. Acad. Sci. USA, 2005, 102, 10813.

[45] L. W. McKeen, Permeability properties of plastics and elastomers, William Andrew, 2016.

[46] H. Ju, B. D. McCloskey, A. C. Sagle, V. A. Kusuma and B. D. Freeman, J. Membrane Sci., 2009, 330, 180.

[47] A. Klinov and I. Anashkin, Processes, 2019, 7, 947.

[48] C. T. Culbertson, S. C. Jacobson and J. M. Ramsey, Talanta, 2002, 56, 365.

[49] M. Arrio-Dupont, S. Cribier, G. Foucault, P. F. Devaux and A. d'Albis, Biophys. J., 1996, 70, 2327.

[50] C. Kotsmar, T. Sells, N. Taylor, D. E. Liu, J. Prausnitz and C. Radke, Macromolecules, 2012, 45, 9177.

[51] G. M. Cruise, D. S. Scharp and J. A. Hubbell, Biomaterials, 1998, 19, 1287.

[52] A. Pluen, P. A. Netti, R. K. Jain and D. A. Berk, Biophys. J, 1999, 77, 542.

[53] E. W. Merrill, K. A. Dennison and C. Sung, Biomaterials, 1993, 14, 1117.

[54] A. G. Ogston, B. Preston and J. Wells, Proceedings of the Royal Society of London. A. Mathematical and Physical Sciences, 1973, 333, 297.

[55] H. Matsuyama, M. Teramoto and H. Urano, J. Membrane Sci., 1997, 126, 151-160.

[56] A. McPherson, Methods, 2004, 34, 254.

[57] S. B. Howard, P. J. Twigg, J. K. Baird and E. J. Meehan, J. Crystal Growth, 1988, 90, 94. 